Revue belge de géographie

Modelling and benchmarking of borders

\title{
Vers une typologie des formes spatiales des limites de l'Europe
}

L'apport des cartes mentales interprétatives d'étudiants

Toward a typology of spatial forms of Europe's boundaries. An approach by students' interpretative mental maps

\section{Sophie de Ruffray}

\section{(2) OpenEdition}

\section{Journals}

Édition électronique

URL : http://journals.openedition.org/belgeo/10627

DOI : $10.4000 /$ belgeo.10627

ISSN : 2294-9135

Éditeur :

National Committee of Geography of Belgium, Société Royale Belge de Géographie

\section{Référence électronique}

Sophie de Ruffray, «Vers une typologie des formes spatiales des limites de l'Europe », Belgeo [En ligne], 1 | 2013, mis en ligne le 31 octobre 2013, consulté le 20 avril 2019. URL : http:// journals.openedition.org/belgeo/10627; DOI : 10.4000/belgeo.10627

Ce document a été généré automatiquement le 20 avril 2019.

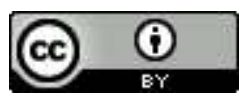

Belgeo est mis à disposition selon les termes de la licence Creative Commons Attribution 4.0 International. 


\title{
Vers une typologie des formes spatiales des limites de l'Europe
}

\author{
L'apport des cartes mentales interprétatives d'étudiants \\ Toward a typology of spatial forms of Europe's boundaries. An approach by \\ students' interpretative mental maps
}

\section{Sophie de Ruffray}

Les recherches menant aux résultats présentés ici ont été financées par le $7^{e ̀ m e}$ programme cadre de recherche et développent de la commission européenne (FP7/2007-2013; grant agreement $n$ - 225260 ; "Visions de l'Europe dans le monde", www.eurobroadmap.eu).

L'auteur tient tout particulièrement à remercier Clarisse Didelon et Nicolas Lambert pour leur aide dans l'élaboration de ce travail ainsi que l'ensemble des partenaires du projet.

1 Afin de questionner la relation entre territoire, frontière et représentation de l'espace, notre approche s'appuie sur l'hypothèse que l'espace européen est un espace subjectif qui reflète non seulement des pratiques de l'espace mais aussi des visions géopolitiques. La frontière, qui peut être considérée comme un sous-ensemble de l'ensemble des limites est marquée par un "processus d'émergence, d'évolution et de stabilisation semblable à celui de n'importe quelle autre limite", mais "plus complexe, plus socialisé et surtout plus enfoncé dans l'historicité" (Raffestin, 1986). Cet objet géographique ne se résume pas seulement à une ligne, limite marquant la séparation entre deux espaces de nature différente, mais bien plus à une zone dotée d'une certaine épaisseur avec la prise en compte des incidences sur l'organisation de l'espace (effets-frontière), la dimension politique (c'est-à-dire qui touche à la structuration d'une société), la dimension symbolique (il est reconnu par un ensemble d'acteurs et sert de marqueur dans l'espace) et la dimension matérielle (qui est inscrite dans le paysage) (Foucher, 2010).

2 L'objectif de cet article est de montrer combien les cartes mentales interprétatives (Didelon et al., 2011) illustrent une typologie des formes spatiales des perceptions des limites. Ainsi la limite de l'Europe n'est pas seulement une frontière -au sens culturel et social que prend la définition de frontier mais aussi boundary avec un sens politique. Les 
limites de l'Europe, de part leurs dimensions politique et symbolique, permettent de proposer une typologie fondée sur les perceptions et les représentations de l'Europe. Dans le cadre plus général des recherches sur l'identité européenne, cet article contribue aux réflexions menées sur le territoire européen et ses délimitations. Plusieurs concepts ont servi de cadre à la réflexion collective de la communauté scientifique pour justifier ou pour remettre en cause la légitimité de certaines frontières (Brennetot et al., 2013). En effet, certains travaux ont souligné les bénéfices liées à l'entrée dans l'Union Européenne (Shöpflin et Wood, 1989 ; Ragaru, 2003) tandis que d'autres ont insisté sur la question des limites en s'interrogeant sur l'implication des élargissements successifs (Boulineau, 2010; Rey et al., 2005), notamment sur les marges (Drevet, 1986, Foucher, 1998). C. Grataloup (2009) met en garde contre le risque de prendre les réflexions du passé comme des évidences acquises et indispensables. De la même façon, le décentrage historique peut s'accompagner d'un décentrage spatial, c'est la position prise dans le projet de recherche européen EuroBroadMap "Visions of Europe in the World", avec l'adoption d'une perspective non eurocentrique (Beauguitte et al., 2012 ; Didelon et al., 2011).

\section{Présentation de l'enquête réalisée dans le cadre du projet Eurobroadmap “Visions de l'Europe dans le monde}

Lors de cette enquête menée à l'échelle internationale dans 43 villes et 18 pays, 10000 étudiants de niveau "licence 3" de six champs disciplinaires (art, santé, sciences de l'ingénieur, géographie, sciences politiques économies) devaient répondre à un questionnaire écrit, dans leurs langues respectives, dont au moins deux questions portaient sur des cartes mentales à deux échelles différentes : une représentant une planisphère en projection polaire pour "dessiner vos propres divisions du monde" (15 au maximum) et une présentant une carte centrée sur l'Europe).

Le choix des pays enquêtés est le reflet de différentes formes de relations vécues à l'égard de l'Union Européenne ou de l'Europe, avec différents niveaux de développement économique et une grande diversité de positionnements géopolitiques à l'échelle mondiale. En effet, certains pays font partie des fondateurs de l'Union Européenne (France, Belgique) ou ont été intégré au cours des élargissements successifs (Portugal, Suède, Hongrie, Malte, Roumanie). D'autres se situent aux marges de l'Union Européenne, déclarés candidats (Turquie), plus ou moins bénéficiaires des politiques de voisinage (Moldavie, Russie). Enfin, certains pays partagent un héritage historique lié aux différentes phases de la colonisation (Brésil, Cameroun, Egypte, Inde, Sénégal, Tunisie) tandis que d'autres ont des relations différentes, et plus indépendantes, vis-à-vis de l'Europe (Azerbaïdjan, (hine).

L'originalité de l'approche menée est fondée non seulement sur une formalisation des représentations mentales de l'Europe, à travers une cartographie interprétative des limites mais aussi par le type d'acteurs interrogés puisqu'il s'agit d'étudiants avec des pratiques spatiales variées, marqués par les connaissances reçues dans le cadre des parcours scolaires et/ou universitaires. Enfin, les choix conceptuels et méthodologiques permettent de mener une approche quantifiée à moyenne échelle qui complète les études de cas, généralement menées, sur les représentations des territoires frontaliers. C'est aussi une manière de montrer combien "la frontière, telle que nous la concevons 
aujourd'hui, doit beaucoup à cette représentation du territoire qu'est la carte" (Rekacewicz, 2008). L'intérêt est de questionner les mécanismes à l'œuvre dans la construction des identités à travers une cartographie sans toutefois pouvoir apprécier d'autres dimensions notamment cognitives parfaitement soulignées par la prise en compte d'une analyse textuelle (Brennetot et al., 2013). Après avoir montré l'intérêt d'utiliser le concept de carte mentale interprétative, une typologie des formes spatiales des limites de l'Europe est proposée pour analyser les perceptions des étudiants à travers les configurations, les tracés des limites et les temporalités.

\section{L'intérêt des cartes mentales interprétatives pour étudier les représentations spatiales}

4 Afin de comprendre la manière dont le monde est perçu par différents types de public, les psychologues ont été les premiers à s'intéresser à l'espace cognitif en posant l'hypothèse que l'espace est chargé de sens et de valeurs. Ces dernières sont propres à chaque individu, mais étudiées collectivement il est possible d'en dégager des traits plus ou moins partagés. Les protocoles mis en place jusqu'à présent avaient pour objectif de capter une perception cognitive de l'espace, plus qu'une interprétation. Notre approche se positionne à la croisée des concepts et des méthodes des cartes mentales dans l'objectif de "capter" l'interprétation d'un espace. Sous le vocable de cartes mentales, sont rassemblées des productions issues du mouvement de "cognitive-behaviourism", né au début des années 1970 aux États-Unis qui mêlait les domaines de la perception et de l'imagination et cherchait à analyser le lien entre représentation et action (Lynch, 1960 ; Gould \& White, 1997 ; Cauvin, 1999 ; Frémont, 1976 ; Moles et Rohmer, 1978).

5 Les "cartes mentales interprétatives", se distinguent par le fait que la consigne posée lors de l'enquête porte non seulement sur un espace, mais sur l'appréciation d'un phénomène dans cet espace. On ne cherche pas à voir comment un individu dessine l'espace, ni quel en est son niveau de connaissance mais comment il circonscrit un phénomène particulier dans un espace dont on lui fournit le fond de carte. L'analyse est plus facile car l'espace "réel" peut être comparé à l'espace "interprété" par les individus enquêtés. Certes, la connaissance et l'affect ne sont sans doute pas absents de la réalisation, mais la délimitation d'un espace sur une carte permet d'en éliminer une partie et diffère de la simple connaissance ou de la projection d'un affect sur un lieu.

Dans l'enquête réalisée dans le cadre du projet Eurobroadmap, une question était intitulée "sur la carte suivante (figure 1), tracez une ligne figurant les contours de l'Europe, selon votre propre point de vue". Pour appréhender une interprétation et éviter toute erreur de localisation et/ou de connaissance, le fond de carte comporte les noms et les tracés des différents pays même s'il introduit des biais de contiguïté pour certains pays, peut-être plus facilement considérés comme "européens" du fait de leur localisation. 
Figure 1. Carte mentale interprétative sur l'Europe - Fond de carte sur lequel les enquêtés traçaient les limites.

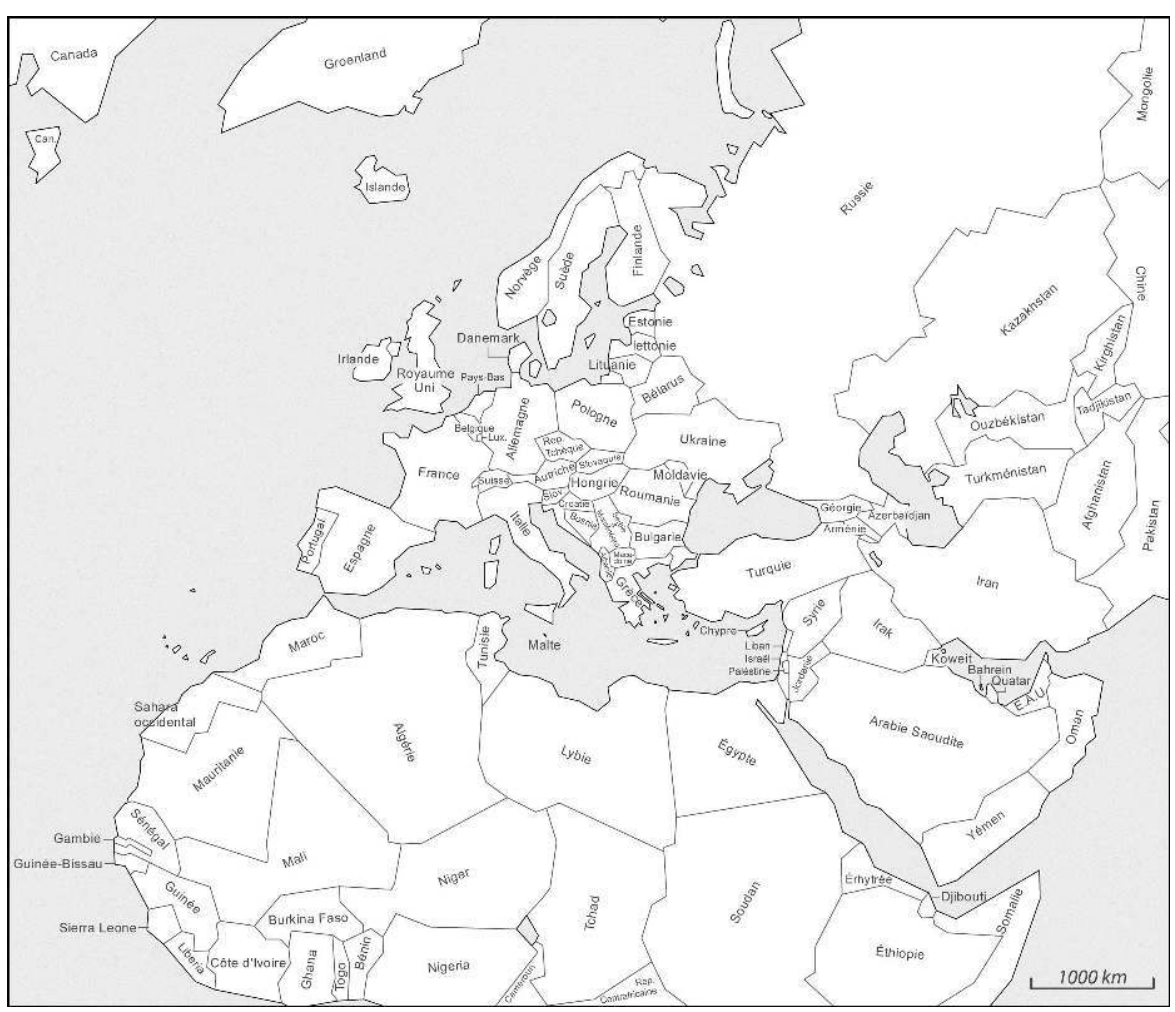

7 Les cartes mentales interprétatives impliquent de prendre comme point de départ de la réflexion, l'espace étudié, qu'il faut appréhender dans sa globalité pour réaliser l'exercice, et non pas l'individu (sa pratique, sa connaissance). Le passage de la représentation individuelle à la représentation collective repose sur l'hypothèse qu'au-delà de la multiplicité des représentations particulières, il existe des mécanismes communs dans la connaissance et la perception. En effet, l'enquêté, même s'il est unique et si sa relation à l'Europe l'est également, est toujours socialisé ce qui suppose une certaine concordance dans ses schémas mentaux et les influences qu'il subit, notamment à travers son parcours scolaire et universitaire.

Pour cartographier et analyser les informations recueillies lors de l'enquête, nous avons recoupé les polygones tracés par les limites d'Europe avec une grille couvrant tout l'espace de la carte. L'objectif est de mettre en évidence l'imprécision introduite par le niveau agrégé, produit par l'ensemble des étudiants alors même que le niveau individuel est une limite nette et claire. (le tracé de la limite de l'Europe est associée à une ligne). La résolution de la grille tient compte de la taille des "petits pays" comme Malte et permet de déterminer le cœur et les marges de l'Europe.

\section{Une configuration de l'Europe à géométrie variable : reflet du processus de la construction européenne}

L'étude de la configuration zonale de la limite européenne, proposée par les résultats de l'enquête, met en évidence les perceptions de l'extension spatiale de l'Europe pour les étudiants interrogés et permet de constater que l'Europe est en grande partie confondue 
avec l'Union Européenne (Didelon et al., 2011), ce qui rejoint les ambiguïtés politiques du premier acteur concerné: l'Union Européenne. L'Europe apparaît comme un objet géographique flou avec des limites plus ou moins tranchées, représentant des frontières plus ou moins acceptées.

10 Ainsi, la carte mentale de la fréquence d'inclusion à l'Europe, montre une configuration auréolaire avec un gradient d'Européanité (Lévy, 1997) et reflète le processus de la construction européenne puisque la graduation correspond aux élargissements successifs de l'Union Européenne.

11 La figure 2 présente une configuration sous la forme d'un cœur où l'appartenance à l'Europe est indiscutable (avec des fréquences de plus de $95 \%$ d'inclusion) et des marges plus ou moins étendues et graduées en fonction des directions.

- le noyau "central européen" est très restreint et correspond aux 6 pays fondateurs de la Communauté Européenne en 1957 avec la Suisse, par effet de contiguïté.

- la première couronne, dont les fréquences d'inclusion à l'Europe sont élevées, correspond à l'extension de l'Union Européenne à la veille de l'élargissement de 2004, avant l'entrée dans l'Union Européenne des pays de l'Europe de l'Est. Le tracé du rideau de fer est persistant avec une discontinuité majeure sur la carte dessinée entre l'Allemagne (réunifiée), l'Autriche et l'Italie d'une part et la Pologne, la République Tchèque et la Slovaquie d'autre part. Par effet de contiguïté, la fréquence d'inclusion observée de la Grèce est comparable alors qu'elle est membre de la Communauté Européenne depuis 1981.

- la dernière couronne qui rassemble l'aire de faible inclusion (fréquences de mois de $5 \%$ de l'échantillon) regroupe les pays du Sud de la Méditerranée et ceux de la Péninsule Arabique qui reflètent aussi l'ambiguïté de la politique de voisinage.

Figure 2. Une vision floue et consensuelle de l'Europe.

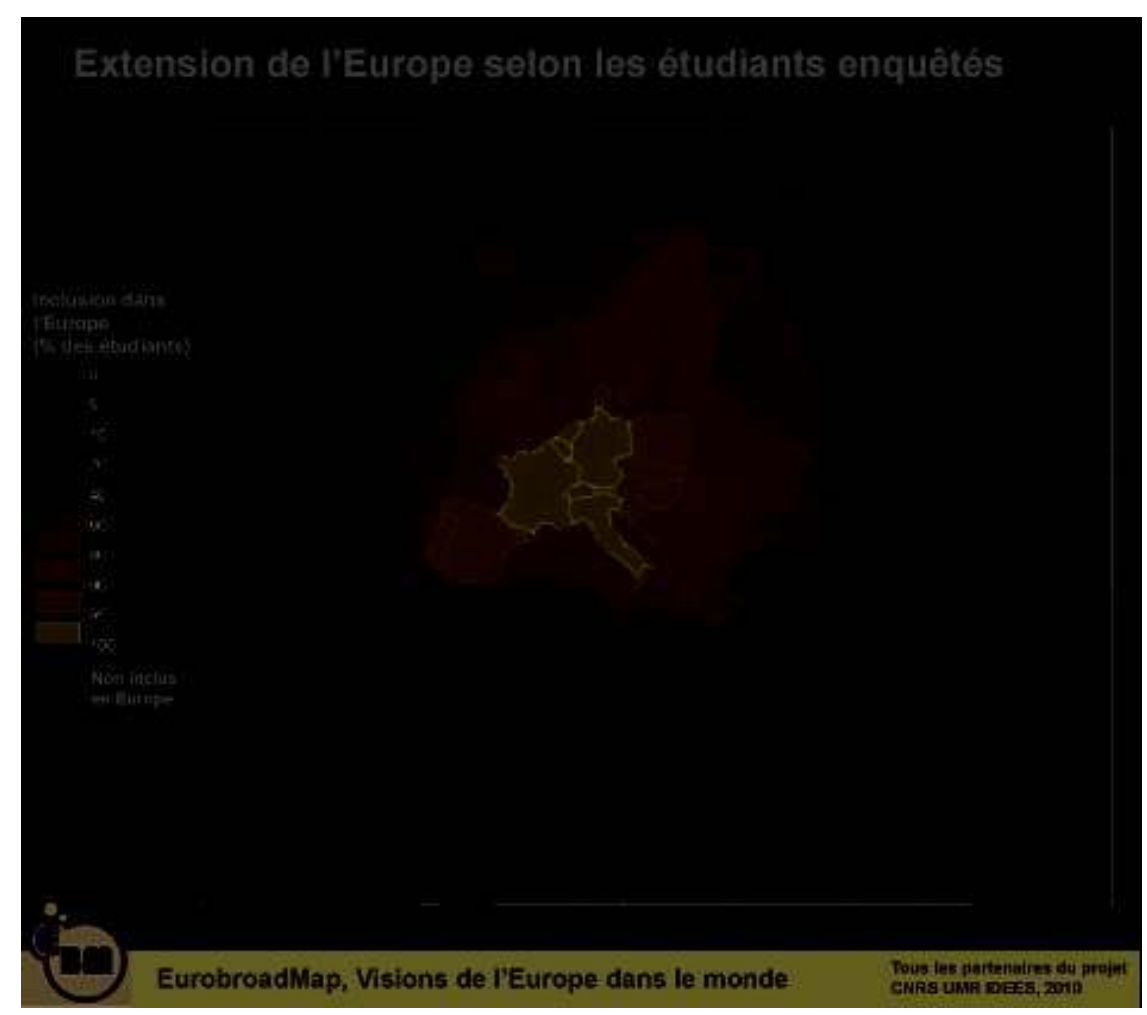

Belgeo, 1 | 2013 

interrogés en 2009 ont suivi des parcours scolaires, avant la réunification. La représentation montre aussi l'ambiguïté de la définition ou plus exactement de l'absence de définition d'un pays "européen". En effet, aucun texte officiel n'a jamais clairement défini ce qu'est un pays "européen", même si des débats sont en cours pour fixer les limites provisoires ou définitives à l'UE. Cette contradiction est inscrite dans les fondements mêmes de la construction politique de l'Union Européenne (Didelon et al., 2011 ; Elissalde, 2009) puisque l'article 237 du traité de Rome stipule que "tout état européen peut devenir membre de la communauté". Les traités d'Amsterdam et de Maastricht poursuivent cette ambiguïté dans la rédaction des articles: "peut devenir membre de l'UE tout Etat européen qui en fait la demande et qui s'engage à respecter les règles en vigueur". De même, les articles concernant l'assemblée parlementaire du Conseil de l'Europe reflètent aussi l'absence de définition. Ainsi, l'article 2 établit que "ne peuvent devenir membres du Conseil de l'Europe que les Etats dont le territoire national est situé en totalité ou en partie sur le continent européen et dont la culture est étroitement liée à la culture européenne". Pour les pays situés au-delà de cette limite, il est possible d'envisager des coopérations, s'il existe des relations culturelles ou d'autres types de relations anciennes, et s'il y a adhésion aux valeurs fondamentales promues par cette organisation". Mais l'article 3 pose que "les frontières n'ont pas encore été complètement définies par le droit international. Dans ces conditions, le Conseil de l'Europe devrait se fonder en principe sur les limites géographiques généralement admises de l'Europe", sans être plus explicite sur leur localisation.

\section{Lignes ou bordures : des limites de l'Europe plus ou moins marquées}

13 La plupart des typologies de frontières utilise le critère de la forme en distinguant des frontières linéaires, des frontières "zones" (Rosière, 2008), privilégiant parfois l'une au détriment de l'autre (Lacoste, 1993 ; Kolossov, 2006, Richard, 2009). Un des intérêts de l'approche par carte mentale est de passer d'une logique individuelle plutôt linéaire (la consigne est de tracer une limite de l'Europe) à une logique collective qui peut prendre les deux formes. Ainsi, une ligne avec des fréquences élevées signifie à la fois une certaine unanimité, symbole de fracture et/ou de différenciation. A l'inverse, lorsque les limites prennent une certaine épaisseur plus ou moins forte, en fonction des fréquences, ces espaces symbolisent des zones d'incertitude significatives d'une appartenance plus ou moins forte à l'Europe, des zones plus ou moins profondes et mouvantes qui correspondent à l'avancée de fronts pionniers.

Ainsi, les limites tracées par les étudiants (figure 3) montrent l'importance du tracé de l'ancien rideau de fer et l'importance de la discontinuité que représente la Méditerranée. Quels que soient les étudiants, cette limite perçue est forte et symbolise à la fois un niveau de développement ou/et un différentiel de niveau socio-économique.

Les configurations diffèrent en fonction des directions :

- la limite Sud suit très nettement la rive sud de la Méditerranée. La discontinuité apparaît très forte au niveau du détroit de Gibraltar, moindre pour celui des Dardanelles, du fait des différences d'inclusion des pays du Nord de la Méditerranée et du rôle de l'altérité perçue vis-à-vis du monde arabo-musulman.

Belgeo, 1 | 2013 
- les niveaux d'inclusion vers l'Ouest avec l'Océan Atlantique et vers l'Est avec la Fédération $\mathrm{du}$ Russie décroissent régulièrement avec les discontinuités des îles d'une part et la définition d'un objet géographique flou d'autre part. Même si cette barrière physique est loin d'être infranchissable, l'Oural semble être une discontinuité symbolique, fortement ancrée dans les esprits, qui a été l'objet d'instrumentalisations diverses au cours de l'histoire. Le nom originel "Zemnoï poyas" qui signifie "ceinture des mondes", évoque l'idée de limites avec un ailleurs différent, voire inquiétant.

Figure 3. Les limites, traceurs de discontinuités.

\section{Limites de l'Europe selon les étudiants enquêtés}

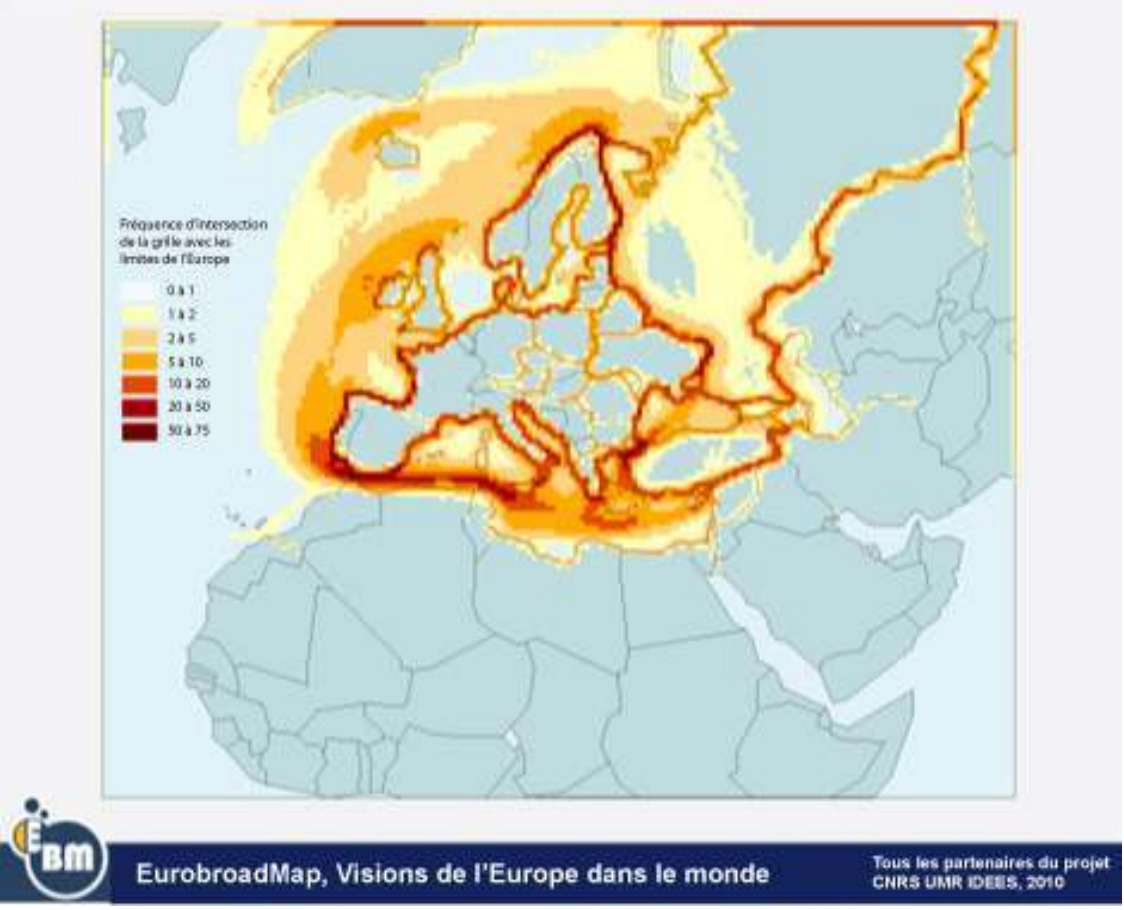

Se distinguent aussi des lignes de fractures internes à l'Union Européenne qui concernent à la fois le Royaume-Uni et l'Irlande, sans doute du fait de la morphologie, mais aussi l'Europe du Nord, avec des pays qui apparaissent plus ou moins fortement identifiés à l'Europe. Au-delà de la ligne de fracture de l'ancien rideau de fer, l'Europe de l'Est apparaît plus morcelé puisque des lignes de faille marquent les cartes mentales, le long des frontières des Etats.

Enfin, des zones d'incertitude apparaissent et concernent particulièrement les Balkans, la Turquie, reflets de la politique de voisinage qui montre bien qu'il n'existe plus une frontière de l'Union Européenne, mais des frontières, plus ou moins complexes, qui concernent à la fois des espaces fonctionnels, des espaces de coopération ou de partenariat (Richard,2009)... Enfin les îles situées au Nord-Ouest ou dans la Méditerranée sont perçues comme des marges européennes plus ou moins identifiées

Au-delà de cette configuration globale représentative de l'échantillon enquêté qui montre une représentation mentale consensuelle de l'Europe, les noyaux présentent des différences importantes. Sur les cartes suivantes (figures 4), la représentation de l'inclusion à l'Europe est présentée dans une perspective de comparaison entre les étudiants des pays de l'Union Européenne, les étudiants des pays voisins et les étudiants 
des pays éloignés. Chaque carte illustre la différence de fréquences d'inclusion de la part des étudiants du pays représenté par rapport à l'ensemble de l'échantillon. Les couleurs jaunes signifient que les lieux concernés ont tendance à être plus inclus en Europe par les étudiants du pays enquêté que par le reste de l'échantillon, le jaune foncé étant significatif après un test statistique du chi2. A l'inverse, lorsque les pays sont en couleur bleue, ils ont été moins fréquemment inclus au sein de l'Europe par le pays enquêté par rapport à l'ensemble. Les pays en gris signifient que les résultats convergent entre les étudiants du pays enquêté et l'ensemble de l'échantillon. Trois seuils d'inclusion sont représentés pour illustrer respectivement la vision restreinte (avec des fréquences de plus de $95 \%$ d'inclusion), la vision moyenne (avec des fréquences de $50 \%$ d'inclusion) et la vision large de l'Europe (avec des fréquences de $5 \%$ d'inclusion).

Les figures $4 \mathrm{a}, 4 \mathrm{~b}$ et $4 \mathrm{c}$ montrent des configurations très proches pour les deux derniers seuils qui correspondent aux limites de l'Union Européenne avec l'Ukraine et la Biélorussie pour la vision moyenne et à un périmètre qui inclut la partie occidentale de la Russie, la Turquie et le Groënland pour la vision large. Quels que soient les seuils, les étudiants n'incluent presque jamais de pays de la rive sud de la Méditerranée même si certains pays enquêtés les ont inclus plus significativement que le reste de l'échantillon (figures $4 \mathrm{a}, 4 \mathrm{~b}, 4 \mathrm{c}$ ). A l'inverse, la configuration du noyau est très différente entre les étudiants des pays membres qui reprennent les frontières de l'Union Européenne avant l'élargissement et les autres pays qui présentent un noyau de petite taille centré généralement sur la France le Benelux et l'Allemagne

Figures 4a. Délimitations de l'Europe pour les étudiants des pays membres de l'Union Européenne: l'exemple de la Belgique et de Malte.

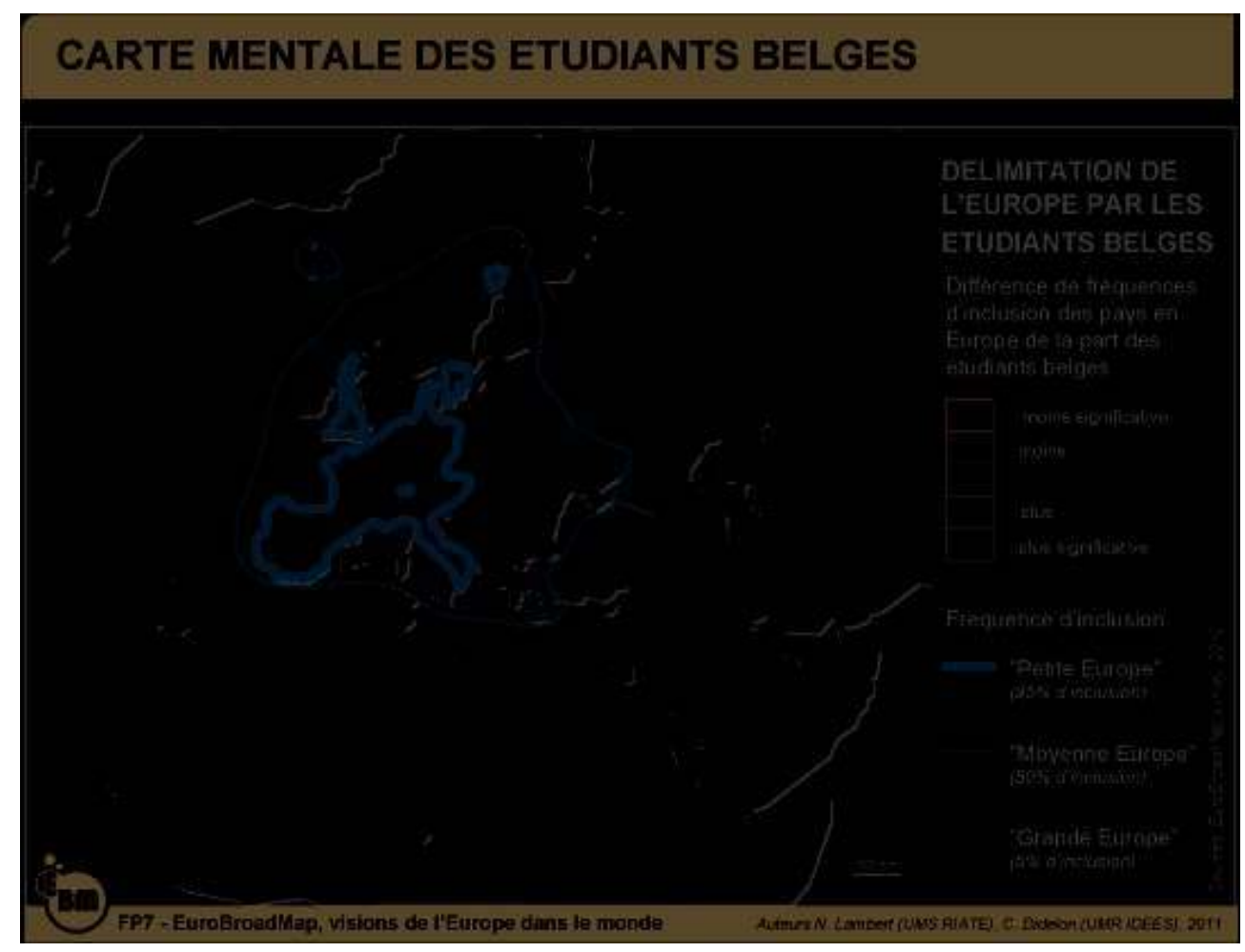




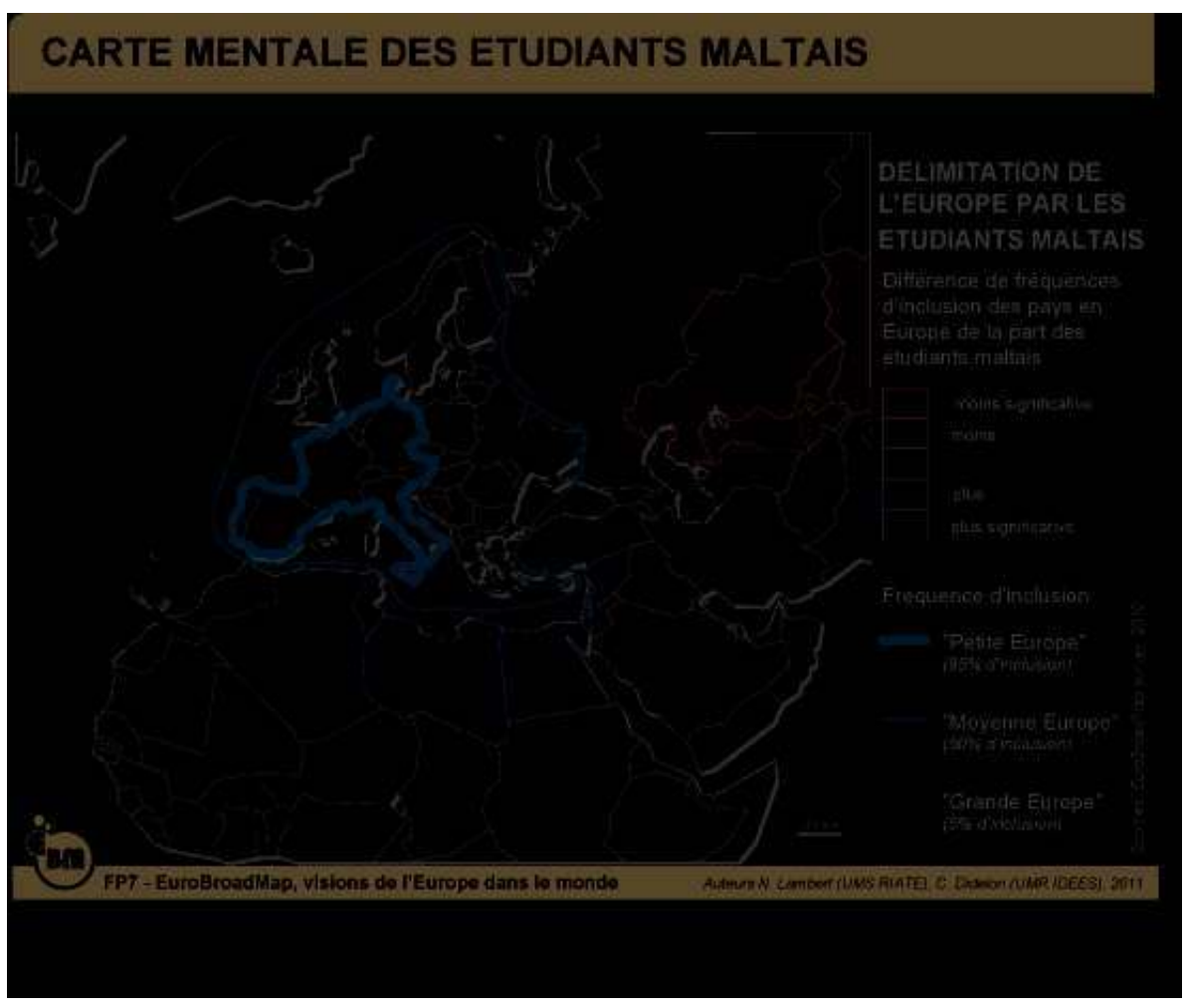

Figures 4b. Délimitations de l'Europe pour les étudiants des pays voisins de l'Union Européenne : l'exemple de la Turquie et de la Russie.

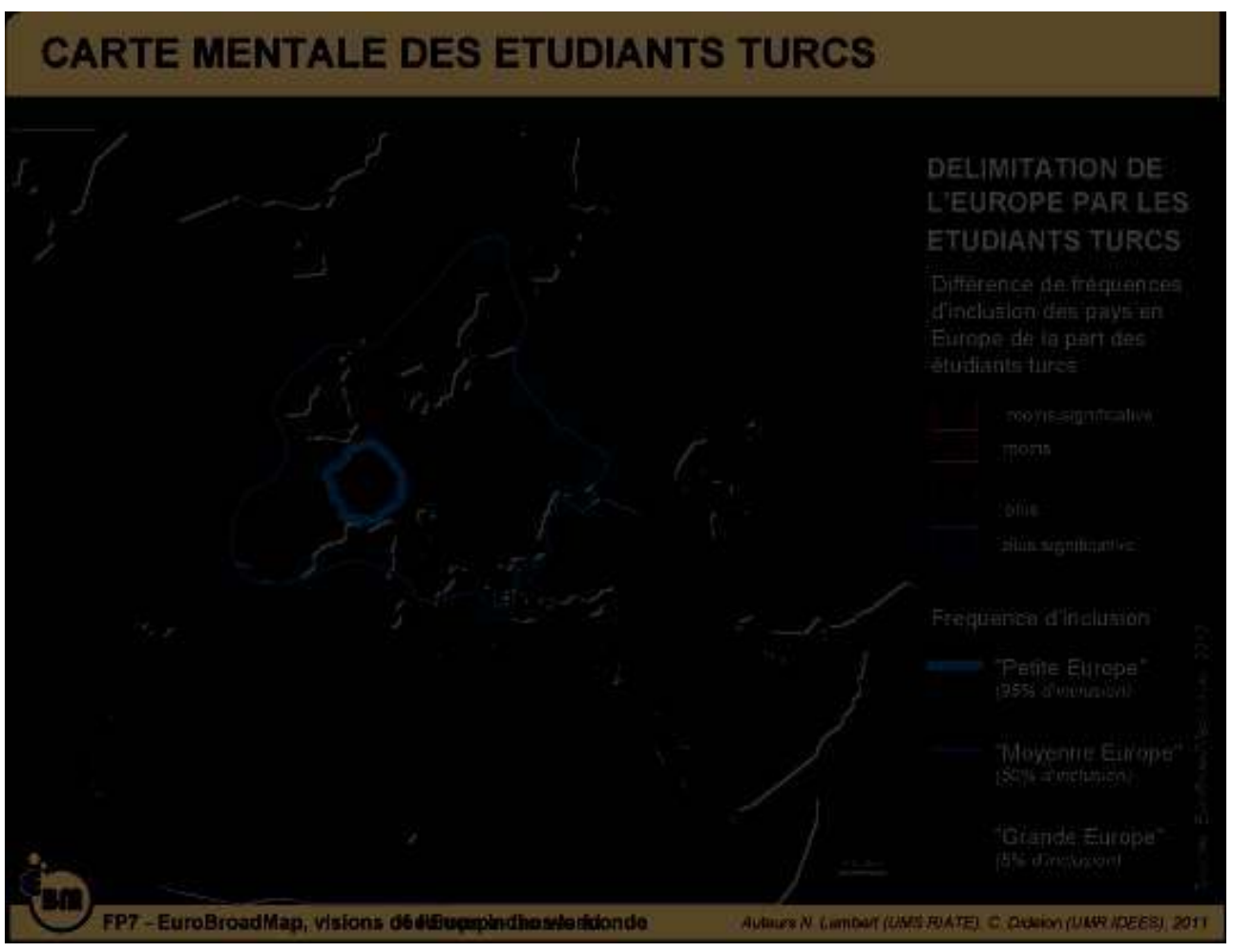




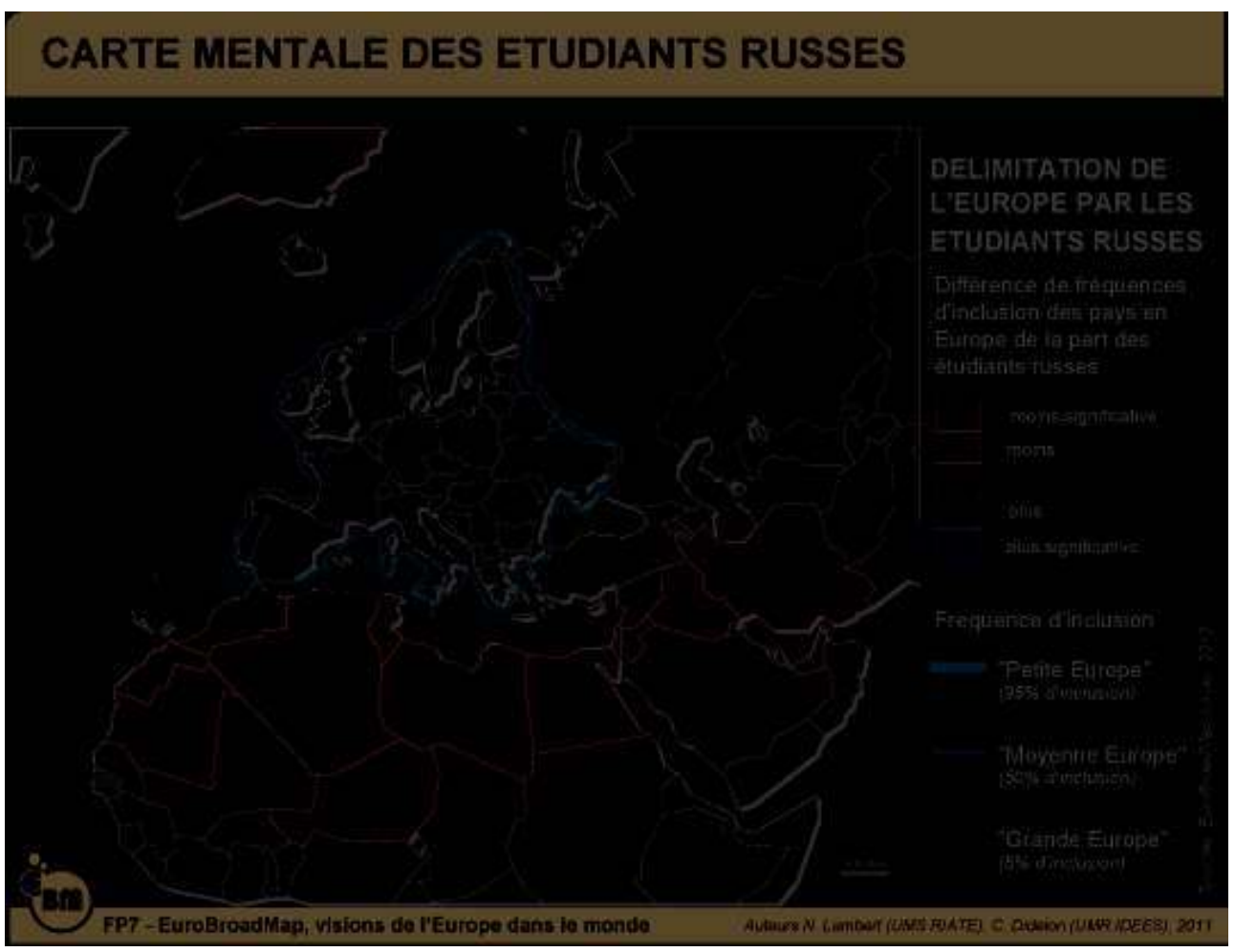

Figures 4c. Délimitations de l'Europe pour les étudiants des pays éloignés : l'exemple du Cameroun et de l'Inde.

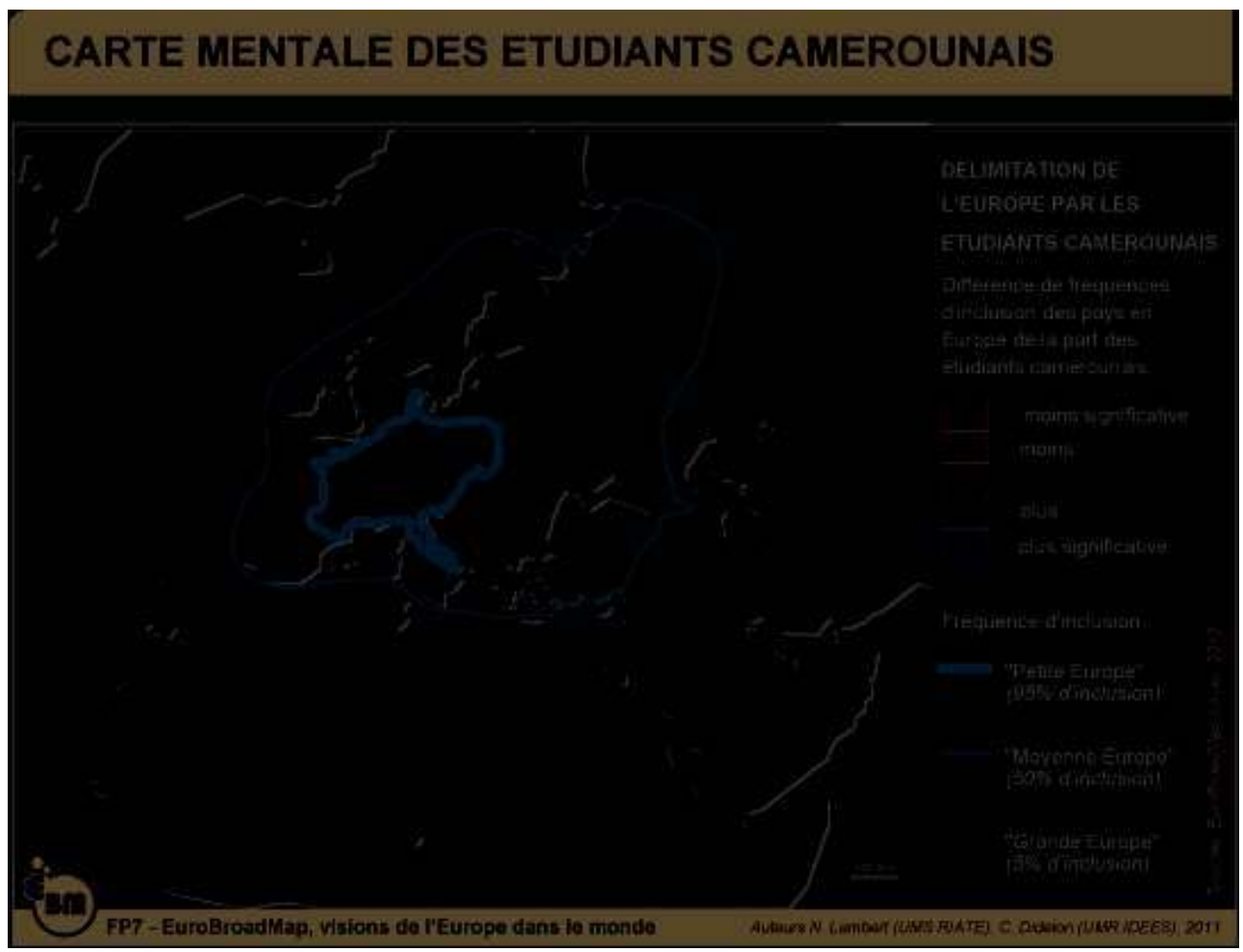




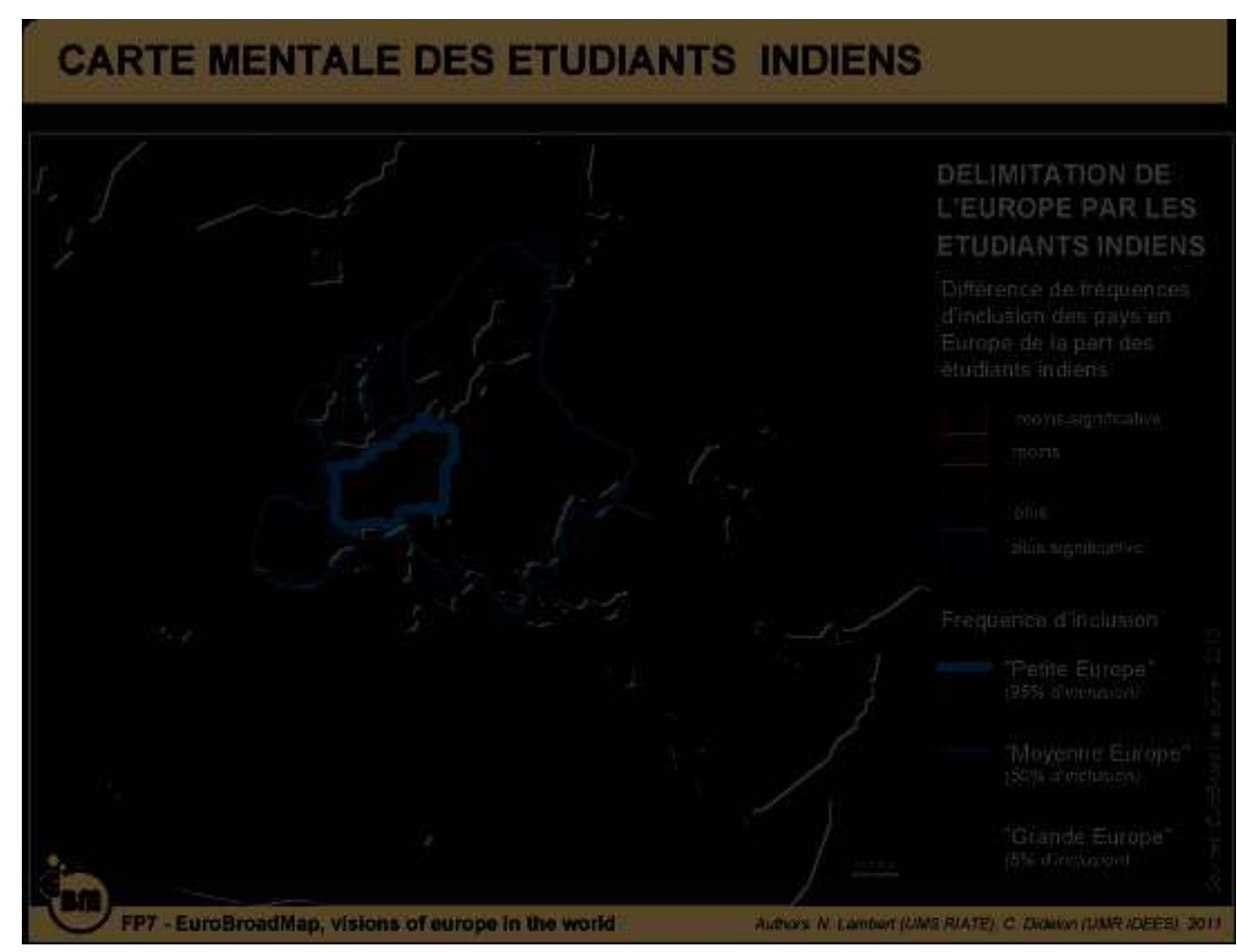

\section{La politique de voisinage de l'Union Européenne, une autre façon d'interpréter les limites à temporalités variables}

Les différents tracés des limites de l'Europe engagent un débat politique et philosophique sur les liens dialectiques qui s'établissent entre territoire précis ("Union Européenne") et un espace imprécis à forte charge symbolique ("l'Europe"). Ainsi, les cartes mentales dessinées par les étudiants retranscrivent non seulement les temporalités du processus de construction européenne mais aussi les ambiguïtés des politiques de voisinages de l'Union Européenne, qui forment une géométrie de plus en plus complexe. Elles représentent aussi un "voisinage perçu", où l'étudiant inclut les pays européens et les pays qui, pour lui, sont "proches" de l'Europe. Ce terme peut à la fois signifier des pays susceptibles d'entrer dans l'Union Européenne ou des pays qui ont des interactions fortes pour des raisons à la fois économiques, sociales, culturelles (commerce, tourisme, migrations, aide au développement...) fondées sur des complémentarités. Un ensemble régional intégré ou en voie d'intégration regroupe l'Union Européenne et ses "voisinages", terme qui recouvre une réalité diversifiée et difficile à définir. Le Conseil Européen de Copenhague (12 et 13 décembre 2002) désigne le terme "voisins" pour les pays proches spatialement de l'Union Européenne. Par la suite, les documents officiels montrent de l'imprécision et de l'incertitude puisqu'ils utilisent les mots "voisin", "voisinage" au singulier et au pluriel. Depuis l'intervention de R. Prodi sur le voisinage de l'Europe élargie en $2003^{1}$, la notion de voisinage s'est institutionnellement stabilisée en incluant les Pays du Caucase (Arménie, Azerbaïdjan, Géorgie), les pays riverains de la Méditerranée et en excluant la Russie et les Balkans occidentaux. En fait, "il faut distinguer plusieurs types de voisinages: un voisinage institutionnel composé des pays concernés par la "Politique Européenne de Voisinage", des voisinages liés à l'Union 
Européenne par des accords différents plus ou moins contraignants et adossés à des aides financières, et un voisinage fonctionnel composé de l'ensemble des pays avec lesquels il existe des interactions sociales, politiques et économiques fortes" (Didelon, et al., 2011).

21 Ainsi, les étudiants incluent très fortement la Suisse qui fait partie du cœur avec les six autres pays fondateurs de l'Union Européenne. De même, la valeur d'inclusion de la Norvège est comparable à celles de l'Irlande, de la Grande Bretagne, de la Finlande, de la Suède et de la Grèce. Pourtant, ces deux pays ont explicitement renoncé par intérêt économique à adhérer à l'Union Européenne (ce qui leur évite de participer aux politiques de cohésion, dont leurs régions ne pourraient guère bénéficier) même s'ils ont signé des accords d'association dans de nombreux domaines qui font d'eux de quasi-membres.

A l'inverse, les représentations mentales incluent au même niveau la Grèce, pays membre de l'Union Européenne depuis 1981, l'Islande et les Balkans, vues par les étudiants, comme un "bloc" voisin alors qu'ils recouvrent des pays avec des statuts très différents (l'adhésion de la Croatie est effective depuis le 1er juillet 2013 ; l'Islande, la Serbie, la Macédoine et le Monténégro ont le statut de pays candidats à l'intégration ; l'Albanie a entamé une demande pour être reconnue candidate ; la Bosnie et le Kosovo ne se sont pas manifestés auprès de l'Union même s'ils sont considérés comme des candidats potentiels). Dans ce cadre, plusieurs de ces entités sont des protectorats qui manquent de viabilité, confrontant l'Union Européenne à un défi pour proposer d'autres modèles, qui ne soient pas des reproductions normatives de ceux conçus pour des Etats du système central (Foucher, 2012). L'Ukraine est perçue comme un pays européen à un degré moindre alors qu'il a ouvert des négociations pour établir un partenariat renforcé avec l'Union Européenne et que l'accord de partenariat comporte des clauses qui le différencient déjà des autres voisins (ouverture d'une zone de libre-échange, accord d'association renforcée). (Delcour L., 2008).

Enfin, la Turquie présente un degré moindre d'inclusion pour les étudiants alors que ce pays est candidat à l'intégration depuis de nombreuses années, et qu'il fait partie de l'Union Douanière européenne. La carte (figure 4B) montre aussi sa représentation en miroir (Yerasimos, 2005). Pour les Turcs, l'Europe persisterait dans l'intention de porter atteinte à la souveraineté et à l'intégrité de la Turquie, résultat des sentiments souverainistes et nationalistes enseignés par leur histoire nationale qui envisage avec réticence tout partage de compétences. Pour les Européens, la Turquie serait incapable de se réformer ou de se démocratiser et oppose sa différence religieuse.

La représentation mentale des étudiants traduit donc l'importance et l'ambiguïté de la notion de voisinage avec la prise en compte partielle des dimensions politiques (pays qui bénéficient de la politique européenne de voisinage), fonctionnelles (zones polarisées par l'Union Européenne) et symboliques (les pays qui dans l'imaginaire de leurs habitants ou dans ceux des habitants de l'Union Européenne en font partie).

La typologie des formes spatiales des perceptions des limites de l' Europe, par une approche subjective que représentent des cartes mentales interprétatives, est riche d'enseignements notamment sur l'appréciation d'un objet politico-géographique mais aussi sur l'intérêt de mener des études à moyenne échelle. Cette approche sur la vision de l'Europe par des étudiants est complémentaire de celles menées sur l'interrogation des fondements du projet européen (Morin, 1987 ; Lévy, 1997 ; Vandermotten et Dézert, 2008). Cette perception résulte autant des expériences individuelles, des imaginaires collectifs mais aussi de médiateurs sociaux (programmes pédagogiques scolaires, presse, 
télévision...). Dans ce cadre, les limites dessinées sur les cartes mentales apparaissent comme des invariants nécessaires, ni négatives, ni positives (Raffestin, 1986). Elles traduisent à la fois des pratiques, des imaginaires mais aussi des reflets des évolutions politico-géographiques admises comme la réunification de l'Allemagne ou à construire comme celles des élargissements successifs de l'Union Européenne. Face à ces représentations mentales qui illustrent les ambiguïtés des acteurs européens, on ne peut que souligner l'intérêt, pour l'Europe, de formuler son projet politique, son dessein économique et son assise territoriale.

\section{BIBLIOGRAPHIE}

AMILHAT-SZARY A.L., FOURNY M.C. (dir.) (2006), Après les frontières, avec la frontière, nouvelles dynamiques transfrontalières en Europe, La Tour d'Aigues, Editions de l'Aube.

ANCEL J. (1938), Géographie des frontières, Paris, Gallimard.

ANDERSON J. (ed.), O’DOWD, L. (ed.), WILSON, T. (ed.) (2003), New Borders of Changing Europe: CrossBorder Cooperation and Governance, London, Taylor \& Francis, 208 p.

BALZACQ T. (2007), "La politique européenne de voisinage, un complexe de sécurité à géométrie variable", Cultures \& Conflits, 66 [En ligne], mis en ligne le 13 mars 2008, consulté le 10 avril 2013, http://conflits.revues.org/2481

BEAUGUITTE L., DIDELON-LOISEAU C. (2013), “L'Europe vue d'ici et d'ailleurs”, L'Espace Politique, 19, 1 [en ligne], mis en ligne le 08 avril 2013, Consulté le 14 avril 2013, http://

espacepolitique.revues.org/index2555.html

BEAUGUITTE L., DIDELON C. et GRASLAND G. (2012), "Le projet EuroBroadMap : visions de l'Europe dans le monde”, Politique européenne, 37, pp. 156-167.

BOUQUET C., VELASCO-GRACIET H. (dir.) (2007), Regards géopolitiques sur les frontières, Paris, L'Harmattan.

BOULINEAU E. (dir.) (2010), “Quelle Europe, vingt ans après la chute du Mur ?", Géocarrefour, 84, 3, Lyon.

BRENNETOT A., EMSELLEM K., GUERIN-PACE F., GARNIER B. (2013), “Dire l'Europe à travers le monde", Cybergeo : European Journal of Geography [En ligne], Politique, Culture, Représentations, document 630, mis en ligne le 24 janvier 2013, consulté le 19 avril 2013, DOI : 10.4000/ cybergeo.25684, http://cybergeo.revues.org/25684

CAUVIN C. (1999), "Propositions pour une approche de la cognition spatiale urbaine", Cybergeo, Revue en ligne de géographie, http://cybergeo.revues.org/5043

DELCOUR L. (2008), "La politique européenne de voisinage et les relations russo-européennes : partenariat stratégique ou lutte d'influence ?", pp. 7-15, Texte disponible sur http://www.etudeseuropeennes.eu/images/stories/Eastern_Partnership/DELCOUR_Laure_FR_FINAL.pdf

DIDELON C., DE RUFFRAY S., GRASLAND C. (2011), Mental maps of the world, EuroBroadmap, Visions of Europe in the world, volumes 1, 2, 3, 
http://halshs.archives-ouvertes.fr/halshs-00654524, http://halshs.archives-ouvertes.fr/ halshs-006654521, http://halshs.archives-ouvertes.fr/halshs-00654523, http://halshs.archivesouvertes.fr/halshs-00654525

DIDELON C., RICHARD Y., VAN HAMME G. (2011), Le territoire européen, Paris, PUF.

DIDELON C., DE RUFFRAY S., LAMBERT N., BOQUET M. (2011), “A World of Interstices: A Fuzzy Logic Approach to the Analysis of Interpretative Maps”, The Cartographic Journal, 48, 2, pp. 100-107.

DREVET J.-F. (1986), La Méditerranée, nouvelle frontière pour l'Europe des Douze ?, Paris, Karthala.

ELISSALDE B. (dir.) (2009), Géopolitique de l'Europe, Paris, Nathan.

FOUCHER M. (1998), Fragments d'Europe. Atlas de l'Europe médiane et orientale, Paris, Fayard.

FOUCHER M. (2010), La bataille des cartes. Analyse critique des visions du monde, Paris, FrançoisBourin.

FOUCHER M. (2012), L'obsession des frontières, Paris, Perrin.

FREMONT A. (1976), La région, espace vécu, Paris, Armand Colin.

GOULD P., WHITE R. (1997), Mental maps ( $2^{\text {nd }}$ edition), Routledge.

GRATALOUP C. (2009), L'Invention des continents, Paris, Larousse.

GUICHONNET P., RAFFESTIN C. (1974), Géographie des frontières, Paris, PUF.

KAFYEKE C. (2006), "L'adhésion de la Turquie à l'Union européenne : enjeux et état du débat", Courrier hebdomadaire du CRISP, 28, 1933-1934, pp. 5-72, www.cairn.info/revue-courrier-hebdomadaire-du-crisp-2006-28-page-5.htm

KOLOSSOV V. (2005), “Etude des frontières, Approches post modernes”, Diogène, 210, pp. 13-27.

LACOSTE Y. (dir.) (2005), “L’Europe et ses limites”, Hérodote, 118, Paris, La Découverte.

LACOSTE Y. (1993), Dictionnaire de géopolique, Paris, Flammarion.

LEVY J. (1997), Europe, une géographie, Paris, Hachette.

LYNCH K. (1960), The image of the city, MIT Press.

MOLES A., \& ROHMER E. (1978), Psychologie de l'espace, Paris, Casterman, collection “Synthèses contemporaines".

RAFFESTIN, C. (1993), “Autour de la fonction sociale de la frontière”, Espace et sociétés, 70-71, pp. 157-164.

RAFFESTIN C. (1986), “Eléments pour une théorie de la frontière”, Diogène, 134, Avril-juin 1986, pp. 3-21.

RAGARU N. (2003), “La Bulgarie et la Roumanie aux portes de l’Union européenne : un si long espoir", Pouvoirs, 106, 3, pp. 99-113.

RATZEL F. (1988), Géographie politique, Paris, Éditions régionales européennes et Economica, (1 $1^{\text {re }}$ éd. en allemand, 1897).

REKACEWICZ P. (2008), “Franchir les frontières : mise en récits et en images. L'exemple des frontières Russie-Union européenne”, http://geoconfluences.ens-lyon.fr/doc/typespace/ frontier/FrontViv.htm

RENARD J.P., (dir.) (1997), Le géographe et les frontières, coll. "Les rendez-vous d'Archimède", L'Harmattan, Paris, 299 p. 
REY V., COUDROY DE LILLE L., BOULINEAU E. (2005), L'élargissement de l'Union Européenne. Réformes territoriales en Europe centrale et orientale, Paris, L'Harmattan.

RICHARD Y. (2009), “L'Union Européenne et ses frontières : un défi aux approches théoriques des frontières”, in ROSIÈRE S. (dir), Penser l'espace politique, Paris, Ellipses.

ROSIERE S. (2008), Dictionnaire de l'espace politique, Paris, Armand Colin.

SCHMID D. (2003), "La Méditerranée dans les politiques extérieures de l'Union européenne : quel avenir pour une bonne idée ?", Revue internationale et stratégique, 1, 49, pp. 23-32.

SCHOPFLIN G., WOOD N. (dir.) (1989), In Search of Central Europe, Cambridge, Polity Press.

VANDERMOTTEN C., DEZERT B. (2008), L'identité de l'Europe : Histoire et Géographie d'une quête d'unité, Paris, Armand Colin.

YERASIMOS S. (2005), “L'Europe vue de la Turquie”, Hérodote, 3, 118, pp. 68-81.

\section{NOTES}

1. Communication de la Commission au Conseil et au Parlement européen. L'Europe élargie Voisinage: un nouveau cadre pour les relations avec nos voisins de l'est et du sud, Bruxelles, 11 mars 2003.

\section{RÉSUMÉS}

Penser l'Europe et ses limites consiste souvent à rechercher une identité européenne, un projet ou des comportements communs dans l'espace. L'approche par les représentations mentales permet de compléter par des perspectives plus subjectives, la perception des frontières. Cet article, réalisé à partir des résultats de l'enquête du projet de recherches Eurobroadmap sur la vision de l'Europe dans le monde permet de mettre en évidence une typologie des formes spatiales des limites de l'Europe. Des cartes mentales interprétatives réalisées par des étudiants montrent une configuration de l'Europe à géométrie variable, à l'image du processus de la construction de l'Union Européenne, avec des limites plus ou moins marquées. Une comparaison entre les cartes mentales des différents pays (membres de l'Union Européenne, pays voisins, pays éloignés...) montre les différences de perceptions de l'Europe.

Thinking about what Europe is, about its limits often consists in trying to identify something such as a European identity, a common project or behavior in space. The approach by mental representations can be supplemented by more subjective perspective, the perception of borders. This article made from the survey results of the research project Eurobroadmap about the vision of Europe in the world allows identifying a typology of spatial forms of the boundaries of Europe. Interpretative mental maps by students show a configuration variable geometry of Europe, as the process of the construction of the European Union, with limits more or less marked. A comparison between the mental maps of different countries (members of the European Union, neighboring countries, remote countries...) shows the differences in perceptions of Europe. 
INDEX

Mots-clés : frontière, Europe, carte mentale, représentation

Keywords : border, mental map

\section{AUTEUR}

SOPHIE DE RUFFRAY

UMR IDEES, Université de Rouen, 7 rue Thomas Becket, 76821 Mont Saint Aignan Cedex, sophie.deruffrayéuniv-rouen.fr 